\title{
Protozoários de vida livre (Ciliophora, Mastigophora e Sarcodia) em dois trechos de um ambiente lótico do nordeste do Brasil e seu potencial uso como bioindicadores
}

\author{
Free-living protozoa (Ciliophora, Mastigophora and Sarcodia) in two stretches of a \\ lotic environment of northeastern Brazil and its potential use as bioindicators
}

\author{
Wellington Sena Lobato Júnior*1 e Magnólia Fernandes Florêncio de Araújo² \\ ${ }^{1}$ Programa de pós graduação em Desenvolvimento e Meio Ambiente-UFRN, Universidade Federal do Rio Grande \\ do Norte, Brasil \\ ${ }^{2}$ Universidade Federal do Rio Grande do Norte,RN, Brasil
}

\begin{abstract}
Resumo
O trabalho teve por objetivo identificar os protozoários de vida livre e caracterizar sua frequência de ocorrência em dois trechos do Rio Pium (P1 e P2), na Bacia Hidrográfica do Rio Pirangi, litoral leste do estado do Rio Grande do Norte, verificando o potencial indicador de qualidade de água desses microrganismos. Foi registrada a presença de 76 táxons de protozoários de vida livre. A técnica de regressão multivariada demonstrou que o nitrogênio total, fósforo total, $\mathrm{pH}$ e o Índice de Estado Trófico estiveram significativamente relacionados $(p \leq 0,05)$ ao número de espécies encontradas no P2. O outro ponto (P1) não apresentou relação significante entre o número de espécies e as variáveis utilizadas no estudo. Os parâmetros físicos, químicos e biológicos pouco interferiram na dinâmica das espécies mais frequentes, caracterizando a maioria como espécies generalistas. Sugere-se que a distribuição dos protozoários de vida livre durante o período de estudo foi dependente da disponibilidade de alimento e pela presença de macrófitas aquáticas nesses ambientes.
\end{abstract}

Palavras-chave: Protozoários de vida livre, ambiente lótico; bioindicadores.

\begin{abstract}
The study aimed to identifying free-living protozoa and characterize their frequency of occurrence in two stretches of the Pium River (P1 and P2) in Pirangi River Basin, east coast of the state of Rio Grande do Norte, verifying the water quality indicator potential of these microorganisms. It was reported the presence of 76 taxa of free-living protozoa. A multivariate regression showed that total nitrogen, total phosphorus, $\mathrm{pH}$ and trophic state index were significantly associated ( $p \leq 0.05$ ) to the number of species found in P2. The other point (P1) showed no significant relationship between number of species and the variables used in the study. The physical, chemical and biological parameters interfered little in the dynamics of the most frequent species, featuring mostly as generalists. It is suggested that the distribution of free-living protozoa during the study period was dependent on the availability of food and the presence of macrophytes in these environments.
\end{abstract}

Keywords: Free-living protozoan; lotic environment; bioindicators. 


\section{Introdução}

A determinação da qualidade da água é um fator de extrema importância no mundo e principalmente no Brasil, que possui uma imensa disponibilidade hídrica quando comparado a outros países. Considerando o litoral do estado do Rio Grande do Norte (RN), que apresenta uma grande demanda turística devido às belezas naturais e clima favorável, tem-se notado uma degradação incisiva dos ambientes lóticos cujas margens são ocupadas sem planejamento (MEDEIROS, 2009). Faz-se necessário o monitoramento e avaliação da qualidade dos corpos aquáticos continentais, que estão suscetíveis à alterações devido fatores naturais ou antropogênicos (PINTO, 2012).

As comunidades de protozoários de vida livre surgem como ferramentas fundamentais no auxílio e determinação da qualidade de água e podem ser usados como bioindicadores robustos em ecossistemas de água doce em rios (JIANG et al., 2007; SHI et al., 2009; TAN et al., 2010; SHI et al., 2012). Os protozoários de vida livre são organismos unicelulares, eucariontes e heterotróficos, havendo, também, representantes mixotróficos (GODINHO e REGALI-SELEGHIM, 1999; SHERR e SHERR, 2002). Estão divididos em três grupos: Ciliophora (ciliados), Mastigophora (flagelados) e Sarcodia (heliozoários; amebas nuas ou com carapaça). Por apresentarem grande diversidade morfológica e fisiológica, sua distribuição mundial é mais limitada pelo habitat do que pelos aspectos geográficos (ROCHA, 2005), mas ainda existe um forte debate quanto à ubiquidade desses organismos (FINLAY e STEBAN, 2001; FINLAY, 2002; FENCHEL e FINLAY, 2004; MITCHELL e MEISTERFELD, 2005; FOISSNER, 2006, 2008), que exercem uma importante função de transferência de energia para níveis tróficos mais elevados na cadeia alimentar aquática por meio do elo microbiano (AZAM et al., 1983).

Pesquisas voltadas para a identificação desses organismos importantes para divulgação de seus resultados para propósitos educacionais, científicos e econômicos (CORLISS, 2001). Além disso, os poucos trabalhos ecológicos que incluem os protozoários geralmente não os identificam ou o fazem de maneira superficial (FOISSNER, 1994). De acordo com Regali-Seleghin et al. (2011), os maiores levantamentos faunísticos de protozoários foram feitos na Europa e América do Norte, e o conhecimento nas outras áreas do planeta é muito pequeno. Existe então uma necessidade de ampliação dos conhecimentos relacionados à diversidade desses organismos, principalmente no Brasil, onde tais estudos ainda são escassos.

Uma das maneiras de intensificar o esforço em estudos taxonômicos de protozoários é por meio do aumento de amostragens em regiões pouco estudadas (MITCHELL e MEISTERFELD, 2005), evidenciando-se características essenciais para diferenciar espécies a serem utilizadas em pesquisas ecológicas, bastante relevantes para os estudos das relações tróficas que permitem a sustentabilidade dos ecossistemas (REGALI-SELEGHIN et al., 2011), e no desenvolvimento de estudos relacionados à educação em ciências (MEDEIROS e ARAÚJO, 2013; ARAÚJO e LOBATO JÚNIOR, 2013).

Diante disso, o presente trabalho teve por objetivo registrar a ocorrência e identificar os protozoários de vida livre em dois trechos de um ecossistema aquático do litoral oriental do RN, numa abordagem que propõe a contribuição para caracterização da distribuição geográfica global de protozoários, com descrição dos indivíduos com alta frequência de ocorrência nos pontos estudados e com o intuito de verificar se as espécies que estão nesses ambientes podem auxiliar na indicação da qualidade da água.

\section{Material e métodos}

\section{1 Área de estudo}

O Rio Pium faz parte da Bacia Hidrográfica do Rio Pirangi (BHP), no litoral leste do Estado do RN, e destacase pelos impactos sofridos por atividades agropecuárias e deposição inadequada de resíduos domésticos em suas margens e atividades recreacionais de balneários. Com aproximadamente $458 \mathrm{~km}^{2}$ - 0,9\% do território estadual esta bacia possui uma grande importância com relação ao abastecimento de água, irrigação, turismo e lazer na região.

\subsection{Amostragem e determinação das variáveis}

Os pontos de amostragem (Figura 1) foram determinados tendo como referência os balneários presentes no Rio Pium, sendo Ponto 1 (P1) localizado antes dos balneários - baixo fluxo de água e com vários bancos de macrófitas - e o Ponto 2 (P2) logo após os balneários.

As coletas ocorreram em dois períodos distintos: um chuvoso (setembro, outubro e novembro) e um seco (março, abril, maio e junho), sendo realizadas apenas na superfície do rio devido à sua baixa profundidade. Os protozoários foram observados sem o uso de fixadores para evitar modificações de suas características morfológicas. A identificação teve base em Patterson (1996), "Protist Information Server" e "Microscope". A frequência de ocorrência foi calculada considerando os táxons mais frequentes com ocorrência maior que $70 \%$.

A temperatura (T), o pH e o oxigênio dissolvido (OD) foram medidos utilizando uma sonda multiparâmetros. As concentrações de clorofila $a(\mathrm{Cl} a)$ foram estimadas segundo Jespersen e Christoffersen (1988) e as análises de nitrogênio total (NT) e fósforo total (FT) seguiram a metodologia de Valderrama (1981). O Índice de Estado Trófico (IET) foi baseado em Lamparelli (2004) para ambientes lóticos. A relação das variáveis ambientais (T, Ph, OD, $\mathrm{Cl} a, \mathrm{NT}, \mathrm{FT}$ ) e o IET foi verificada na análise da diversidade de protozoários. 


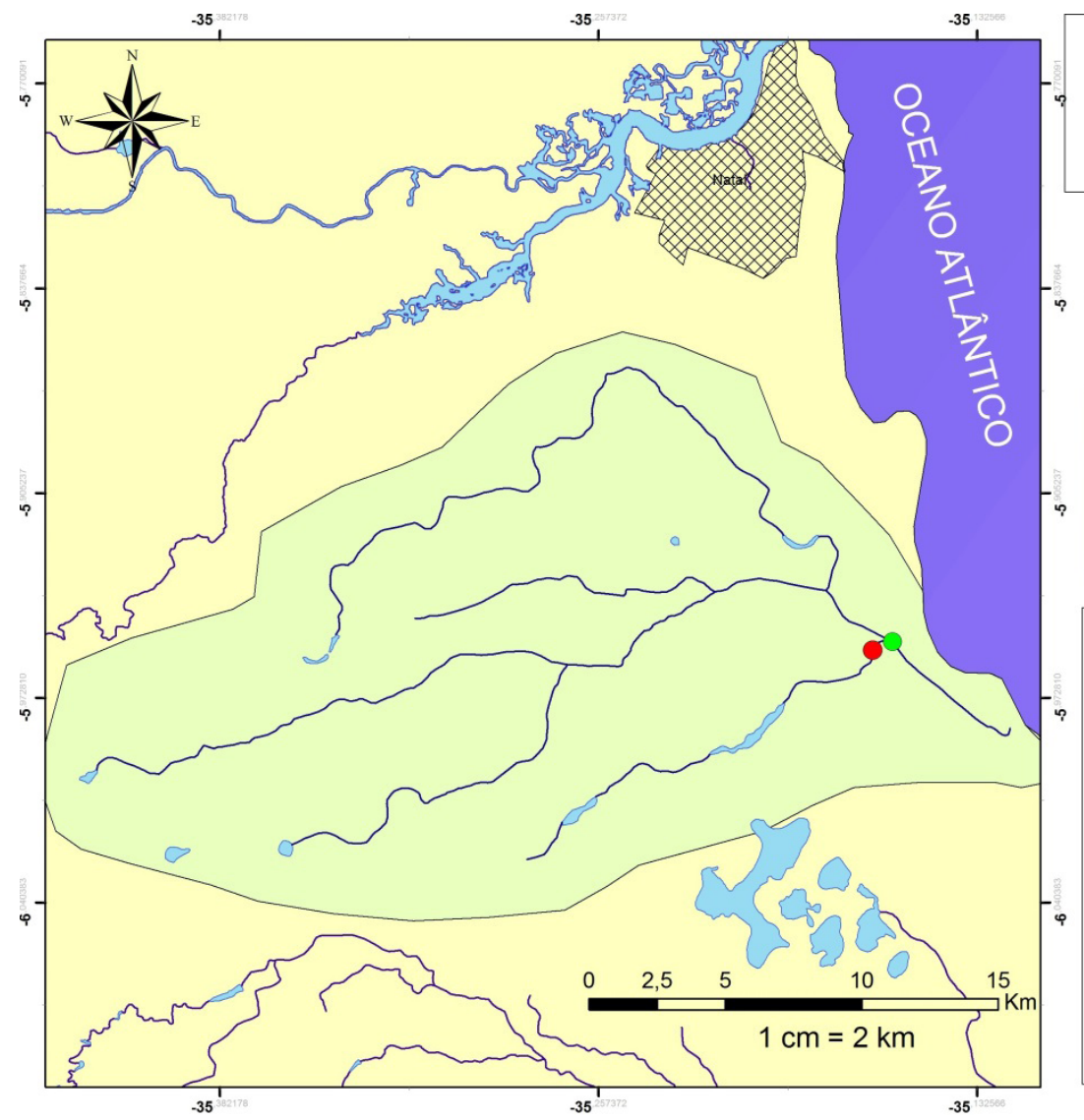

\section{BACIA HIDROGRÁFICA PIRANGI}

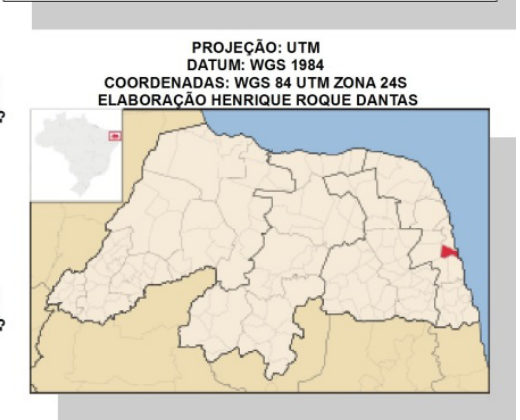

\section{LEGENDA}

- P 1

- $\quad \mathrm{P} 2$

\section{Reservatórios/Lagoas \\ Bacia Hidrográfica Pirangi \\ Capital Estadual \\ Limite RN \\ Oceano atlântico}

Figura 1. Bacia do Rio Pirangi e pontos de amostragem - Rio Pium. P1: Ponto 1; P2: Ponto 2.

\section{Resultados e discussão}

Os valores das variáveis ambientais referentes ao período de coleta são mostrados na Tabela 1. A temperatura da água foi o parâmetro mais constante durante o período de amostragem em ambos os pontos. No entanto, diferenças significativas $(p<0,05)$ foram encontradas entre os dois períodos em outros parâmetros. Em P1 apenas o nitrogênio total apresentou variação significativa entre o período seco e o período chuvoso. Em P2, diferenças entre os dois períodos foram encontrados tanto para o nitrogênio total quanto para $\mathrm{Cl} a$.

Tabela 1. Variáveis ambientais no Rio Pium (P1 e P2) durante o período de amostragem.

\begin{tabular}{|c|c|c|c|c|c|c|c|c|c|c|c|c|c|}
\hline & & \multicolumn{6}{|c|}{ P1 } & \multicolumn{6}{|c|}{ P2 } \\
\hline & DATA & $\begin{array}{c}\mathrm{T} \\
\left({ }^{\circ} \mathrm{C}\right)\end{array}$ & $\begin{array}{c}\mathrm{Cl} a \\
\left(\mu \mathrm{g} \mathrm{L}^{-1}\right)\end{array}$ & $\mathrm{pH}$ & $\begin{array}{c}\text { NT } \\
\left(\mu \mathrm{g} \mathrm{L}^{-1}\right)\end{array}$ & $\begin{array}{c}\text { FT } \\
\left(\mu \mathrm{g} \mathrm{L}^{-1}\right)\end{array}$ & $\begin{array}{c}\mathrm{OD} \\
\left(\mathrm{mg} \mathrm{L}^{-1}\right)\end{array}$ & $\begin{array}{c}\mathrm{T} \\
\left({ }^{\circ} \mathrm{C}\right)\end{array}$ & $\begin{array}{c}\mathrm{Cl} \mathbf{a} \\
\left(\mu \mathrm{g} \mathrm{L}^{-1}\right)\end{array}$ & $\mathrm{pH}$ & $\begin{array}{c}\text { NT } \\
\left(\mu g \mathrm{~L}^{-1}\right)\end{array}$ & $\begin{array}{c}\text { FT } \\
\left(\mu \mathrm{g} \mathrm{L}^{-1}\right)\end{array}$ & $\begin{array}{c}\mathrm{OD} \\
\left(\mathrm{mg} \mathrm{L}^{-1}\right)\end{array}$ \\
\hline \multirow{3}{*}{ 号 } & set/11 & 28,6 & 3,0 & 6,5 & 323 & 3,66 & 4,60 & 27,4 & 0,00 & 6,50 & 2260 & 5,80 & 5,49 \\
\hline & out/11 & 28,5 & 7,7 & 6,4 & 1243 & 8,60 & 4,52 & 27,8 & 0,00 & 6,67 & 780 & 9,50 & 5,60 \\
\hline & nov/11 & 29,5 & 23,6 & 6,5 & 1373 & 23,8 & 4,50 & 28,9 & 4,08 & 6,82 & 713 & 16,80 & 5,63 \\
\hline \multirow{4}{*}{ 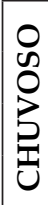 } & $\operatorname{mar} / 12$ & 30,1 & 23,3 & 6,7 & 234 & 69,6 & 4,61 & 28,5 & 3,72 & 6,93 & 657 & 37,10 & 5,76 \\
\hline & $\mathrm{abr} / 12$ & 29,4 & 22,7 & 6,8 & 599 & 23,00 & 4,74 & 28,3 & 3,99 & 6,89 & 685 & 38,00 & 5,74 \\
\hline & mai/12 & 29,1 & 8,4 & 6,8 & 282 & 17,10 & 4,73 & 28,7 & 3,12 & 7,00 & 533 & 19,60 & 5,79 \\
\hline & jun/12 & 28,5 & 8,4 & 6,8 & 253 & 2,83 & 4,82 & 27,3 & 3,12 & 7,00 & 560 & 7,00 & 5,94 \\
\hline \multicolumn{2}{|r|}{ Média } & 29,1 & 13,9 & 6,6 & 615,3 & 21,22 & 4,65 & 28,1 & 2,57 & 6,83 & 884 & 19,11 & 5,71 \\
\hline \multicolumn{2}{|r|}{ D.P. } & 0,60 & 8,9 & 0,18 & 490,16 & 22,99 & 1,93 & 0,63 & 1,79 & 0,18 & 612,74 & 13,55 & 1,97 \\
\hline
\end{tabular}

Nota: $\mathrm{T}=$ Temperatura, $\mathrm{Cl}$ a = Clorofila a, NT = Nitrogênio Total, FT = Fósforo Total, OD = Oxigênio Dissolvido, D.P. = Desvio Padrão 
Tabela 2. Frequência de ocorrência dos táxons de protozoários identificados em P1 e P2 durante o período de coleta.

\begin{tabular}{|c|c|c|c|c|c|}
\hline Táxons & \multicolumn{2}{|c|}{$\begin{array}{c}\text { Ocorrência } \\
(\%)\end{array}$} & \multirow{2}{*}{\begin{tabular}{|l|} 
Táxons \\
Mastigophora (cont.) \\
\end{tabular}} & \multicolumn{2}{|c|}{ Ocorrência (\%) } \\
\hline Ciliophora & P1 & $\mathbf{P 2}$ & & P1 & P2 \\
\hline Aspidisca aculeata & 42,85 & 57,14 & Euglena granulata & 0 & 14,28 \\
\hline Aspidisca cicada* & 71,42 & 14,28 & Euglena mutabilis & 14,28 & 0 \\
\hline Aspidisca steini & 14,28 & 0 & Euglena sp. & 14,28 & 14,28 \\
\hline Chilodonella sp. & 14,28 & 0 & Euglena spyrogira & 14,28 & 14,28 \\
\hline Chlamydodon sp. & 14,28 & 0 & Sp. 1 & 14,28 & 28,57 \\
\hline Glaucoma frontata & 0 & 14,28 & Peranema sp. & 0 & 28,57 \\
\hline Climacostomum sp. & 14,28 & 14,28 & Petalomonas sp. & 0 & 14,28 \\
\hline Sp. 3 & 14,28 & 0 & Phacus longicauda & 14,28 & 0 \\
\hline Cinetochilum margaritaceum ${ }^{*}$ & 85,71 & 85,71 & Phacus pleuronectes & 0 & 14,28 \\
\hline Cohnilembus sp. & 14,28 & 0 & Phacus torta & 0 & 42,85 \\
\hline Coleps hirtus & 42,85 & 42,85 & Phacus triqueter & 0 & 14,28 \\
\hline Colpoda sp. & 0 & 14,28 & Trachelomonas armata & 42,85 & 0 \\
\hline Cyclidium sp. & 0 & 14,28 & Trachelomonas sp. $1^{*}$ & 85,71 & 85,71 \\
\hline Dileptus amphileptoides & 0 & 14,28 & Trachelomonas sp. 2 & 14,28 & 0 \\
\hline Dileptus anser & 14,28 & 0 & Sarcodia & & \\
\hline Euplotes woodruffi & 28,57 & 0 & Actinosphaerium sp. & 42,85 & 57,14 \\
\hline Halteria grandinella & 14,28 & 42,85 & Amoeba proteus & 57,14 & 42,85 \\
\hline Lembadion sp. & 42,85 & 28,57 & Amoeba radiosa & 0 & 14,28 \\
\hline Litonotus cygnus & 0 & 14,28 & Arcella hemisphaerica & 14,28 & 0 \\
\hline Litonotus sp. 1 & 28,57 & 14,28 & Arcella sp. 1 & 0 & 14,28 \\
\hline Litonotus sp. 2 & 0 & 14,28 & Arcella sp. 2 & 0 & 14,28 \\
\hline Litonotus sp. 3 & 14,28 & 0 & Centropyxis sp. & 14,28 & 0 \\
\hline Mesodinium sp. & 28,57 & 0 & Difflugia globulosa & 28,57 & 42,85 \\
\hline Paramecium caudatum & 42,85 & 42,85 & Difflugia pristis & 57,14 & 57,14 \\
\hline Paramecium multimicronucleatum & 14,28 & 0 & Difflugia pyriformis & 14,28 & 42,85 \\
\hline Paramecium trichium & 14,28 & 28,57 & Difflugia sp. & 14,28 & 0 \\
\hline Pleuronema sp. & 14,28 & 28,57 & Euglypha acanthophora & 0 & 28,57 \\
\hline Stentor sp. & 42,85 & 0 & Euglypha rotunda & 14,28 & 0 \\
\hline Stentor coeruleus & 42,85 & 42,85 & Euglypha tuberculata & 57,14 & 42,85 \\
\hline Strobilidium sp. & 14,28 & 14,28 & Hartmanella sp. & 14,28 & 0 \\
\hline Urocentrum turbo & 57,14 & 0 & Mayorella bigemma & 28,57 & 14,28 \\
\hline Uroleptus sp. & 28,57 & 42,85 & Mayorella Leidyi & 14,28 & 14,28 \\
\hline Vorticella sp. & 42,85 & 42,85 & Mayorella sp. & 0 & 14,28 \\
\hline Mastigophora & & & Sp. 2 & 14,28 & 0 \\
\hline Anisonema acinus & 0 & 28,57 & Trinema complanatum & 14,28 & 14,28 \\
\hline Anisonema sp. & 14,28 & 57,14 & Trinema lineare & 28,57 & 28,57 \\
\hline Bodo saltans & 57,14 & 42,85 & Trinema enchelys* & 71,42 & 28,57 \\
\hline Entosiphon sulcatum ${ }^{*}$ & 71,42 & 71,42 & Vannella sp. 1 & 14,28 & 14,28 \\
\hline Euglena anabaena & 14,28 & 0 & Vannella sp. 2 & 14,28 & 28,57 \\
\hline
\end{tabular}

Nota: *Táxons mais frequentes $(\geq 70 \%)$. 
O NT atingiu maiores concentrações no período de estiagem: $1373 \mu \mathrm{g} \mathrm{L}-1 \mathrm{em}$ P1 e $2260 \mu \mathrm{g} \mathrm{L}{ }^{-1}$ em P2. As concentrações de $\mathrm{Cl} a$ obtiveram os maiores valores durante período seco em ambos os pontos, havendo diferenças significativas entre os dois pontos. As concentrações foram maiores em P1e, assim, as concentrações de $\mathrm{Cl} a$ foram cruciais para determinação do IET.

Um total de 76 protozoários foi encontrado durante todo o período de coleta, compreendendo 33 ciliados, 19 flagelados e 24 sarcodíneos (Tabela 2). Apesar da proximidade entres os pontos de amostragem, dos 76 táxons identificados, apenas 35 (40,05\%) ocorreram em ambos os pontos. Foram registrados 23 táxons exclusivamente em P1 e 18 em P2. A ocorrência de ciliados apresentou diferenças qualitativas entre os pontos estudados, tendose observados 21 táxons em P1 e 27 em P2. Já os flagelados e sarcodíneos apresentaram diferenças menores entre os pontos coletados. Dentro dos flagelados, foram catalogados 12 e 15 táxons em P1 e P2 respectivamente. O grupo Sarcodia foi representado por 20 táxons em P1 e 18 en P2.

Trabalhos indicam que quanto maior a diversidade de protozoários, melhor a qualidade da água (XU et al., 2008; JIANG et al., 2011). No entanto, apesar de sofrer menos impactos, P1 mostrou-se mais eutrofizado e com maior diversidade que P2. Lamparelli (2004) afirma que águas com baixa correnteza e profundidade, característico de $\mathrm{P} 1$, tendem a apresentar maior sedimentação do material em suspensão, possibilitando uma maior transparência e consequentemente uma maior produtividade. Esse quadro mostra que as espécies nesse ponto coexistem sem dominância a partir dos nutrientes disponíveis.

O P2 apresentou níveis de NT maiores que P1 e níveis de $\mathrm{Cl} a$ bem inferiores. Segundo Esteves (1998), em nível de sedimento, as bactérias são muito importantes para a fixação total de nitrogênio de um sistema aquático, e o fornecimento de energia por meio dos detritos geralmente excede àquela produzida pela fotossíntese. Em sedimentos sobrecarregados de matéria orgânica, as bactérias e a matéria orgânica dissolvida são as principais fontes de alimento, logo, P2 pode ter apresentado uma maior quantidade de bactérias, ocasionando uma baixa diversidade pela exclusão competitiva entre espécies de protozoários com hábitos alimentares semelhantes - bacterívoros (PATTERSON, 1996). Aspidisca Cicada, Chilodonela sp., Euplotes woodruffi, Lembadion sp., Mesodinium sp., Stentor sp., Urocentrum turbo, Bodo saltans e Trinema enchelys são alguns exemplos de protozoários bacterívoros que apresentaram ocorrência menor ou ausente em P2, provavelmente devido a maior competição entre as espécies, quando comparado a P1.

Os padrões das comunidades de protozoários nas sete amostras exibiram uma clara sucessão temporal relativa à composição de espécies em ambos os pontos estudados. O aumento da quantidade de táxons em ambos os ambientes durante o período chuvoso pode ter sido ocasionado pela maior disponibilidade de nutrientes trazidos pela chuva, diminuindo a competição entre as espécies e aumentando a diversidade. Paramecium caudatum foi registrado no período seco (setembro e outubro) e no final do período chuvoso em P1 (maio), e durante todo o período seco em P2. Trata-se de um ciliado heterotrófico que se alimenta principalmente de bactérias, fazendo parte de comunidades bentônicas com excessivo teor de carga orgânica - polissapróbico e $\boldsymbol{\alpha}$-mesossapróbico (FOISSNER e BERGER, 1996; PATTERSON, 1996). Assim, é possível assumir uma melhora da qualidade da água de ambos os ambientes durante o período chuvoso.

Percebe-se que a ocorrência dos protozoários, principalmente de ciliados, em ambos os pontos parece estar mais relacionada com a disponibilidade de alimento do que com os níveis tróficos do ambiente. Ao estudar a distribuição de protozoários em ambientes lênticos e lótico em ambientes tropicais, Medeiros et al. (2013) também chegaram a conclusão semelhante, descartando os níveis tróficos como responsáveis pela distribuição dos protozoários em ocasiões particulares.

A maior frequência de amebas testadas em P1 pode ser explicada pela maior quantidade de macrófitas aquáticas nesse ponto. Em estudos desenvolvidos em ambientes tropicais, Araújo e Godinho (2008) e Medeiros et al. (2013) observaram existir uma associação entre amebas testadas e macrófitas aquáticas em ambientes lóticos. Isso caracteriza estes protozoários como pertencentes principalmente a ambientes lóticos (LANSAC-TOHA et al., 2000) e exclui a dependência desses organismos em relação aos níveis tróficos dos ambientes. Actinosphaerium $s p$. foi o único representante do grupo Heliozoa, estando presente tanto em P1 quanto P2. Leonov (2010) relata que organismos deste grupo são amplamente distribuídos e ocorrem em ambientes com diferentes características.

Com base na análise realizada com os dados do Rio Pium observou-se que o NT, FT, pH e o IET foram significantes $(\mathrm{p} \leq 0,05)$ para o número de espécies encontradas em P2, demonstrando que alterações acentuadas das variáveis físico-químicas podem influenciar a diversidade de táxons de protozoários de vida livre.

Cinco táxons obtiveram larga frequência de ocorrência, aparecendo em mais de 70\% das amostras e em ambos os pontos de amostragem: Aspidisca cicada, Cinetochilum margaritaceum, Entosiphon sulcatum, Travhelomonas sp. e Trinema enchelys. Organismos com ocorrências elevadas podem ser considerados como importantes componentes da rede trófica (ARAÚJO e COSTA, 2007). Apenas A. cicada destacou-se como possível bioindicador. Tratase de uma espécie geralmente encontrada em águas paradas, como em P1, que apresenta baixo fluxo de água e profundidade, alimentando-se principalmente de bactérias e fazendo parte da comunidade perifítica e bentônica. A. cicada é um organismo $\beta$-mesossapróbio e $\alpha$-mesossapróbio, presente em ambientes com teor de carga orgânica moderada e forte, respectivamente (FOISSNER e BERGER, 1996). 


\section{Conclusão}

O levantamento pioneiro da biodiversidade taxonômica dos protozoários de vida livre em trechos da bacia Hidrográfica do Rio Pirangi representa uma contribuição para o conhecimento da distribuição desses organismos em ambientes tropicais. Nesse ambiente, estudos sobre a comunidade bacteriana são necessários para melhor aferição a respeito da diferença de trofia nos dois pontos estudados. Os parâmetros físicos, químicos e biológicos pouco interferiram na dinâmica das espécies mais frequentes, caracterizando a maioria como espécies generalistas.

Estudos comparativos entre ambientes lóticos e lênticos nesta bacia podem fornecer informações mais eficazes no que diz respeito à identificação das variáveis que controlam a dinâmica desses organismos. Em futuros estudos, a caracterização dos protozoários de vida livre deverá ir além de observações e medições morfológicas, uma vez que fica clara a necessidade do uso de microscopia eletrônica e análises moleculares na identificação de um grupo tão amplo e disperso quanto este, para a indicação de diferentes impactos.

\section{Agradecimentos}

Welington Lobato Júnior agradece à CAPES pela bolsa concedida; e ao CONSULEST (Departamento de Estatística - UFRN) pelas análises estatísticas realizadas.

\section{Referências}

ARAÚJO, M. F. F.; COSTA, I. A. S. Comunidades microbianas (bacterioplâncton e protozooplâncton) em reservatórios do semi-árido brasileiro. Oecologia Brasiliensis, v. 11 n. 3, p. 422-432, 2007.

ARAÚJO, M. F. F.; GODINHO, M. Spatial and Seasonal variations of plantonick protists (Mastigophora, Sarcodina and Ciliophora) in a river-lacustrine system in northeast Brazil. Acta Limnologica Brasilie, v. 20, n. 3, p. 235-244, 2008.

ARAÚJO, M. F. F.; LOBATO JÚNIOR, W. S. Percepção sobre protozoários no ensino fundamental: um diagnóstico em escolas de uma região litorânea do Nordeste brasileiro. Acta Scientiae, v. 15, n. 2, p. 339-347, 2013.

AZAM, F.; FENCHEL, T.; FIELD, J. G.; GRAY, J. S.; MEYER-REIL, L. A.; THINGSTAD, F. The ecological role of watercolumn microbes in the sea. Marine Ecology Progress, n. 10, p. 257-263, 1983.
CORLISS, J. O. Have the Protozoa Been Overlooked? BioScience, v. 51, n. 6, p. 424 - 425. 2001.

ESTEVES, F. A. Fundamentos de Limnologia. 2 ed. Rio de Janeiro: Interciência. 1998. 602 p.

SHERR, E. B.; SHERR, B. F. Significance of predation by protists in aquatic microbial food webs. Antonie van Leeuwenhoek, n. 81, p. 293-308, 2002.

FENCHEL, T.; FINLAY, B. J. The Ubiquity of Small Species: Patterns of Local and Global Diversity. BioScience, v. 54, n. 8, p. 777-784, 2004.

FOISSNER, W. Progress in taxonomy of planktonic freshwater ciliates. Marine Microbial Food Webs, n. 8, p. 9-35, 1994.

FOISSNER, W.; BERGER, H. A user-friendly guide to the ciliates (Protozoa, Ciliophora) commonly used by hydrobiologists as bioindicators in rivers, lakes and waste waters, with notes on their ecology. Freshwater Biology, n. 35, p. 275-482, 1996.

FOISSNER, W. Biogeography and Dispersal of Microorganisms: A Review Emphasizing Protists. Acta Protozoologica, n. 45, p. 111-136, 2006.

FOISSNER, W. Protist diversity and distribution: some basic considerations. Biodiversity \& Conservation, n. 17, p. 235-242, 2008.

FINLAY, B. J.; ESTEBAN, G. F. Ubiquitous microbes and ecosystem function. Limnetica, v. 20, n. 1, 31-43, 2001.

FINLAY, B. J. Global Dispersal of Free-Living Microbial Eukaryote Species. Science, n. 296, p. 1061-1063, 2002.

GONG, J.; SONG, W.; WARREN, A. Periphytic ciliate colonization: annual cycle and responses to environmental conditions. Aquatic Microbial Ecology, n. 39, p. 159-170, 2005.

GODINHO, M. J. L.; REGALI-SELEGHIM, M. H. Diversidade no reino protista: protozoários de vida livre. In: Microrganismos e Vírus. São Paulo, 1999, p. 84-91.

JESPERSEN, A. M.; CHRISTOFFERSEN, K. Measurements of chlorophyll-a from phytoplankton using ethanol as extraction solvent. Archives of Hydrobiology, n. 109, p. 445-454, 1988.

JIANG, J.; WU, S.; SHEN, Y. Effects of seasonal succession and water pollution on the protozoan community structure in a eutrophic lake. Chemosphere, n. 66, p. 523-532, 2007. 
JIANG, Y., XU, H.; AL-RASHEID, K. A. S.; WARREN, A.; HU, X. Planktonic ciliate communities in a semienclosed bay of Yellow Sea, northern China: annual cycle. Journal of the Marine Biological Association of the United Kingdom, v, 91, n. 1, p. 97-105, 2011. doi:10.1017/S002531541000175X.

KISS, Á.K.; ÁCS, É.; KISS, K.T.; TÖRÖK, J.K. Structure and seasonal dynamics of the protozoan community (heterotrophic flagellates, ciliates, amoeboid protozoa) in the plankton of a large river (River Danube, Hungary). European Journal of Protistology, n. 45, p. 121-138, 2009.

LAMPARELLI, M. C. Grau de trofia em corpos d'água do Estado de São Paulo: avaliação dos métodos de monitoramento, 2004, 238f. Tese (Doutorado em Ciências na Área de Ecossistemas Terrestres e Aquáticos) - Instituto de Biociências, Universidade de São Paulo, São Paulo, 2004.

LANSAC-TÔHA, F. A.; VELHO, L. F. M.; ZIMMERMANNCALLEGARI, M. C.; BONECKER, C. C. On the occurrence of testate amoebae (Protozoa, Rhizopoda) in Brazilian inland waters. I. Family Arcellidae. Acta Scientiarum, v. 22, n. 2, p. 355-363, 2000.

MEDEIROS, J. R. Influência das águas da Bacia Hidrográfica Pirangi na Balneabilidade das praias de Pirangi, nos municípios de Nísia Floresta e Parnamirim - Rio Grande do Norte, Brasil, 2009, 122f. Dissertação (Mestrado em Engenharia Sanitária) - Universidade Federal do Rio Grande do Norte, Natal, 2009.

MEDEIROS, M. L. Q.; ARAÚJO, M. F. F.; NETO, L. S.; AMORIM, A. S. Spatial and temporal distribution of free-living protozoa in aquatic environments of a Brazilian semi-arid region. Revista Ambiente e Água - An Interdisciplinary Journal of Applied Sciences, v. 8, n. 2, 2013. doi: 10.4136/ambi-agua.1129.

MEDEIROS, M., L. Q.; ARAÚJO, M. F. F. Protozoários, qualidade de água dos açudes e doenças de veiculação hídrica na percepção de professores e alunos de escolas públicas do ensino básico. Educação Ambiental em Ação, ano 12, n. 45, out. 2013. Disponível em.< http:// www.revistaea.org/artigo.php?idartigo $=1621$ \&class $=21>$. Acesso em: 10 out. 2013.

MICROSCOPE. 2012. In: Browse organisms alphabetically. Disponível em: <http://starcentral.mbl.edu/microscope/ portal.php>. Acesso em: 02 set. 2011.

MITCHELL, E.A.D.; MEISTERFELD, R. Taxonomic confusion blurs the debate on cosmopolitanism versus local endemism of free living protists. Protist, n. 156, p. 263-267, 2005.
PATTERSON, D. J. Free-living freshwater protozoa: a colour guide. New York: J. Wiley \& Sons, 1996. 223 p.

PEREIRA, A. P. S.; DO VASCO, A. N.; BRITTO, F. B.; JÚNIOR, A. V. M.; GARCIA, C. A. B.; NOGUEIRA, E. M. $S$. Estudo da diversidade da comunidade tecamebiana (protozoa: rhizopoda) na sub-bacia hidrográfica do Rio Poxim-SE. Scientia Plena, v. 7, n. 4, p. 1-9, 2011.

PROTIST INFORMATION SERVER. Digital specimen archives. In: Japan Science and Technology Corporation (JST). Disponível em: $<$ http://protist.i.hosei.ac.jp/protist_ menuE.html>. Acesso em: 02 set. 2011.

PINTO, A. J.; LOVE, N. G. Bioreactor Function under Perturbation Scenarios Is Affected by Interactions between Bacteria and Protozoa. Environmental Science \& Technology, n. 46, p. 7558-7566, 2012.

REGALI-SELEGHIM, M.H.; GODINHO, M.J.L.; MATSUMURA-TUNDISI, T. Checklist of "protozoans" from São Paulo State, Brazil. Biota Neotropica, v. 11, n. 1, p. 135-172, 2011.

ROCHA, O. Águas Doces. In: Avaliação do estado do conhecimento da diversidade biológica do Brasil. Brasília: MMA, 2005, p. 15-52.

SHI, X.; TAN, X.; LIU, G.; YANG, X.; ZHANG, Z.; LIU, X.; NIE, P. Seasonal changes of phytoplankton community and biological evaluation about water quality of an artificial lake in Northeast China. Journal of Hangzhou Normal University, n. 8, p. 132-142, 2009.

SHI, X.; LIU, X.; LIU, G.; SUN, Z.; XU, H. An approach to analyzing spatial patterns of protozoan communities for assessing water quality in the Hangzhou section of Jing-Hang Grand Canal in China. Environmental Science and Pollution Research, n. 19, p. 739-747, 2012.

TAN, X.; SHI, X.; LIU, G.; XU, H.; NIE, P. An approach to analyzing taxonomic patterns of protozoan communities for monitoring water quality in Songhua River, northeast China. Hydrobiologia, n. 638, p. 193-201, 2010.

VALDERRAMA, J. C. The simultaneous analysis of total nitrogen an total phosphorus in natural waters. Marine Chemistry, n. 10, p. 109-122, 1981.

XU, H.; SONG, W.; WARREN, A.; AL-RASHEID, K. A. S.; AL-FARRAJ. S. A.; GONG, J.; HU, X. Planktonic protist communities in a semi-enclosed mariculture pond: structuralvariation and correlation with environmental conditions. Journal of the Marine Biological Association of the United Kingdom, v. 88, n. 7, p. 1353-1362, 2008. 